\title{
Yet another proof of Hawking and Ellis's Lemma 8.5.5
}

\author{
S. Krasnikov*
}

\begin{abstract}
The fact that the null generators of a future Cauchy horizon are past complete was first proved by Hawking and Ellis [1]. Then Budzyński, Kondracki, and Królak outlined a proof free from the error found in the original one [2]. Finally, Minguzzi has recently published his version of the proof [3] patching a previously unnoticed hole in the preceding two. I am not aware of any flaws in that last proof, but it is quite difficult. In this note I present a simpler one.
\end{abstract}

\section{Introduction}

Let $H^{+}$denote a future Cauchy horizon. A lemma by Hawking and Ellis says

Lemma 8.5.5 of [1]. If $H^{+}(\mathcal{Q})$ is compact for a partial Cauchy surface $\mathcal{Q}$, then the null geodesic generating segments of $H^{+}(\mathcal{Q})$ are geodesically complete in the past direction.

The lemma itself has never been doubted (to my knowledge), but the proof offered in [1] was found to be flawed, see [3] and references therein. To improve this situation - which is important, because the lemma is a popular tool in mathematical relativity - Minguzzi has recently published a new, more accurate, proof of the lemma (or, to be precise, of some strengthening of it).

In this comment I present yet another proof of the same fact. The reason for doing this is that my version is much simpler (partly because its major part is replaced by a reference to a lemma proved elsewhere).

\footnotetext{
*Email: S.V.Krasnikov@mail.ru
} 


\section{The proposition and its proof}

In a spacetime $M$ consider a past inextendible null curve $\gamma$ totally imprisoned in a compact set $\mathcal{K}$. Pick a smooth unit timelike future directed vector field $\boldsymbol{\tau}$ on $M$ and define (uniquely up to an additive constant) the "arc length parameter" $l$ on $\gamma$ by the requirement

$$
g\left(\partial_{l}, \boldsymbol{\tau}\right)=-1
$$

In addition to $l$ define on $\gamma$ an affine parameter $s$ so that $\partial_{s}$ is future directed and $s=0$ at $l=0$. Now $\gamma$ is characterized by the (evidently negative) function

$$
h \equiv g\left(\partial_{s}, \boldsymbol{\tau}\right)
$$

which relates $l$ to $s$ :

$$
h=-\frac{\mathrm{d} l}{\mathrm{~d} s}, \quad s(l)=\int_{l}^{0} \frac{\mathrm{d} \breve{l}}{h(\breve{l})} .
$$

As is proven in [4]

$$
h^{\prime} / h \text { is bounded on } \gamma \text {. }
$$

Further, a few minor changes - a past inextendible $\gamma(l)$ with $l \in(-\infty, 0]$ instead of the future inextendible $\gamma(l)$ with $l \in[0, \infty)$ and an arbitrary compact $\mathcal{K}$ instead of some specific $\mathcal{L}$ - leave [4, Lemma 8] valid while bringing it to the following form.

Lemma 8 of [4]. Assume $h(l)$ is such that there exists a smooth function $f(l)$ defined at non-positive $l$ and obeying for some non-negative constants $c_{1}, \underline{f}, \bar{f}$ the inequalities

$$
\underline{f} \leq f \leq \bar{f}, \quad\left|f^{\prime} / f\right|<\infty
$$

and

$$
h^{\prime} / h<-f^{\prime} / f-c_{1} f, \quad \forall l \leq 0 .
$$

Then there is a timelike past inextendible curve $\gamma_{\varkappa_{0}}$ which is obtained by moving each point of $\gamma$ to the past along the integral curves of $\boldsymbol{\tau}$ and which is totally imprisoned in a compact set $\mathcal{O}$.

Proposition. If $\mathcal{K}$ is a subset of the boundary of a globally hyperbolic past set $M^{\text {in }}$, then $\gamma$ is past complete. 
Proof. Suppose the lemma is false and $\gamma$ is past incomplete. This would mean that the affine parameter $s$ is bounded from below and, correspondingly, the integral (2) converges at $l \rightarrow-\infty$. Which allows one to define the following smooth positive function on $(-\infty, 0]$

$$
f(l) \equiv \frac{1}{h}\left[-\int_{l}^{0} \frac{\mathrm{d} \breve{l}}{h(\breve{l})}+2 \int_{-\infty}^{0} \frac{\mathrm{d} \breve{l}}{h(\breve{l})}\right]^{-1} .
$$

$f$ so defined satisfies the equation

$$
f^{\prime} / f+h^{\prime} / h=-f
$$

and consequently, condition (4) holds.

As $h$ is negative, the boundedness of the integral (2) provides a simple estimate

$$
\infty>-\int_{-\infty}^{0} \frac{\mathrm{d} \breve{l}}{h(\breve{l})}>-\frac{1 / h(l)}{\left(\left|h^{\prime} / h\right|\right)^{\max }}, \quad \forall l \in(-\infty, 0]
$$

which implies, due to (3), that $1 / h$ is bounded. It follows then from (5) that $f$ is bounded too. Finally, the just proven boundedness of $f$ combined with (6) and (3) implies the boundedness of $f^{\prime} / f$. Thus all the conditions of Lemma 2 are fulfilled and the corresponding variation transforms $\gamma$ into a past inextendible timelike curve $\gamma_{\varkappa_{0}}$. The latter being timelike lies entirely in the closed (due to the globally hyperbolicity of $M^{\text {in }}$, to which $\gamma_{\varkappa_{0}}(0)$ belongs) set $J^{-}\left(\gamma_{\varkappa_{0}}(0)\right) \subset M^{\text {in }}$ (the inclusion follows from the fact that $M^{\text {in }}$ is a past set). Thus, $\gamma_{\varkappa_{0}}$ is totally imprisoned in the compact subset $\mathcal{O} \cap J^{-}\left(\gamma_{\varkappa_{0}}(0)\right)$ of the globally hyperbolic spacetime $M^{\text {in }}$, which is forbidden by [1, proposition 6.4.7].

\section{References}

[1] S. W. Hawking and G. F. R. Ellis The Large scale structure of spacetime (Cambridge, Cambridge University Press, 1973).

[2] R. Budzyński, W. Kondracki, A. Krolak New properties of Cauchy and event horizons, arXiv:gr-qc/0011033. 
[3] E. Minguzzi, J. Math. Phys. 55, 082503 (2014).

[4] S. Krasnikov, Physical Review D 90, 024067 (2014). 